\title{
PRIMEROS REGISTROS ESTATALES DE ESPECIES MEXICANAS DE STAPHYLINIDAE (INSECTA: COLEOPTERA)
}

\author{
JuAN MÁRQUEZ \& JuLIETA ASIAIN \\ Laboratorio de Sistemática Animal, Centro de Investigaciones Biológicas, Universidad Autónoma del \\ Estado de Hidalgo, km 4.5 carretera Pachuca-Tulancingo s/n, Ciudad Universitaria, Col. Carboneras, \\ 42184 Mineral de la Reforma, Hidalgo, México.<jmarquez@uaeh.edu.mx>, <asiainae@yahoo.com>
}

Márquez, J. \& J. Asiain. 2012. Primeros registros estatales de especies mexicanas de Staphylinidae (Insecta: Coleoptera). Acta Zoológica Mexicana (n. s.), 28(1): 118-132.

RESUMEN. Se presentan 14 primeros registros estatales de 13 especies de Staphylinidae para los estados de Hidalgo (siete especies), San Luis Potosí (cinco especies) y Guanajuato (dos especies). Se incluyen los datos de distribución geográfica hasta ahora conocidos para cada especie, los cuales se ubicaron en las provincias biogeográficas mexicanas. Al analizar la distribución geográfica de cada especie, se apreció que corresponden con cuatro patrones de distribución, tres de ellos previamente reconocidos en distintos grupos de coleópteros y uno más que se reconoció en este análisis: Componente Mexicano de Montaña (una especie), Componente Mesoamericano (siete especies), Componente Mexicano de Montaña-Componente Neártico Continental (tres especies) y patrón biogeográfico Sureste de México (dos especies). Además de ubicar a las 13 especies en cada uno de estos patrones, se incluyen otras especies de Staphylinidae y de varias familias de coleópteros que sustentan estos patrones.

Palabras clave: Staphylinidae, biogeografía, México, primeros registros.

Márquez, J. \& J. Asiain. 2012. First state records for Mexican species of Staphylinidae (Insecta: Coleoptera). Acta Zoológica Mexicana (n. s.), 28(1): 118-132.

ABSTRACT. Fourteen first state records of 13 species of Staphylinidae from Hidalgo (seven species), San Luis Potosí (five species) and Guanajuato (two species) are presented. Geographical distribution data currently known for each species are included, which were located in the Mexican biogeographic provinces. The geographical distribution analysis of each species suggests a correspondence with four distribution patterns, three of them previously recognized in several beetle groups and one more identified in this analysis: Mountain Mexican Component (one species), Mesoamerican Component (seven species), Mountain Mexican Component-Continental Nearctic Component (three species), and Southeastern Mexican (two species). In addition to assign the 13 species to each pattern, other species of Staphylinidae and species of several families supporting these patterns were included.

Key words: Staphylinidae, biogeography, Mexico, first records.

Recibido: 17/03/2011; aceptado: 25/10/2011. 


\section{INTRODUCCIÓN}

En los últimos diez años se ha incrementado el conocimiento biológico sobre las especies mexicanas de Staphylinidae, permitiendo ubicar a Veracruz, Oaxaca y Chiapas como los estados con el mayor número de especies (Navarrete-Heredia \& Zaragoza-Caballero 2006), aspecto que coincide en lo general con otros grupos de coleópteros, como Scarabaeoidea (Delgado \& Márquez 2006). A pesar de este aumento en el estudio de los estafilínidos mexicanos, aún es reducida la información que existe sobre ellos, ya que pocos grupos se pueden identificar a nivel de especies por la carencia de literatura adecuada y se desconoce mucho sobre aspectos como su demografía, estacionalidad, preferencias de hábitat y tipos de alimentos, etc. El conocimiento sobre su distribución geográfica es solo parcial, ya que para la mayoría de las especies se tienen pocos registros, muchos de ellos históricos, que no representan información cercana a la realidad, principalmente por la carencia de colectas, y cuando éstas existen, por la falta de identificación específica de los ejemplares y el análisis de sus registros.

Los datos de distribución geográfica de las especies de Staphylinidae de México se han utilizado en distintos estudios con enfoques biogeográficos, como los de Márquez \& Asiain (2006), quienes analizaron los patrones de distribución de los estafilínidos mexicanos con base en datos de distribución de 670 especies de las más de 1500 conocidas hasta entonces en el país. Márquez $(2004,2006)$ y Asiain et al. (2010) aportaron nuevos datos de distribución geográfica de estafilínidos mexicanos, analizando de manera general y desde una perspectiva biogeográfica histórica, a qué patrones corresponden estas distribuciones. También se han incluido algunas especies de estafilínidos en análisis biogeográficos, junto con especies de otras familias de coleópteros (Márquez \& Morrone 2004; Morrone \& Márquez 2001).

El objetivo de esta contribución es proporcionar 14 primeros registros estatales de especies mexicanas de Staphylinidae y discutir los patrones de distribución que presentan con respecto a patrones biogeográficos previamente documentados.

\section{MATERIAL Y MÉTODOS}

Los ejemplares analizados se encuentran depositados en la Colección de Coleoptera de la Universidad Autónoma del Estado de Hidalgo (CC-UAEH). Fueron identificados utilizando literatura especializada y por comparación con organismos previamente identificados y depositados en la colección ya señalada, excepto Bledius inornatus que fue identificado por L. Herman, especialista en Oxytelinae. Los datos de distribución geográfica se obtuvieron de la literatura disponible. Se anotan en orden alfabético los estados de México con registros, separando otros países con registros mediante punto y coma; los datos de localidades por estado se anotan entre paréntesis adelante del estado correspondiente, excepto cuando la información existente carece 
de estos datos. Los mapas de distribución de las especies (Figs. 1-4) sólo muestran los registros para México.

\section{RESULTADOS}

Las siguientes 13 especies representan los 14 primeros registros estatales:

1. Micropeplinae: Pleplomicrus mexicanus Campbell, 1978 (Fig. 1a). Hidalgo: "México: Hidalgo, La Misión, Lomas del Pericón, bosque mesófilo de montaña, 1377 m, N $21^{\circ} 06^{\prime} 46.0^{\prime \prime}, \mathrm{W} 99^{\circ} 06^{\prime} 15.6^{\prime \prime}$, trampa de intercepción de vuelo, 8 a 16-III-2008, J. Márquez y J. Asiain cols.”(2). Registros previos: Chiapas (Tuxtla Gutiérrez) y Veracruz (Huatusco) (Campbell 1978; Santiago Jiménez 1999).

2. Oxytelinae, Deleasterini: Deleaster trimaculatus Fall, 1910 (Fig. 1b). Hidalgo: "México: Hidalgo, Omitlán de Juárez, bosque de encino, $2407 \mathrm{~m}, \mathrm{~N} 20^{\circ}$ 9' 45.5", W $98^{\circ} 38^{\prime}$ 3.9", bajo roca y hojarasca de riachuelo, 29-III-2003, J. Márquez y J. Asiain cols." (2). Registros previos: Chihuahua (Creel, Cuiteco); USA (Navarrete-Heredia et al. 2002).

3. Oxytelinae, Thinobiini: Bledius inornatus Sharp, 1887 (Fig. 2a). Hidalgo: "México: Hidalgo, Chapulhuacán, Arroyo Blanco, bosque tropical subperennifolio, $263 \mathrm{~m}, \mathrm{~N} 21^{\circ} 09^{\prime} 38^{\prime \prime}, \mathrm{W} 99^{\circ} 00^{\prime} 01^{\prime \prime}$, trampa de luz, 21-X-2006, J. Márquez, J. Asiain, F. Ramírez y L. Herman cols." (2). Registros previos: Tabasco (Teapa); Costa Rica y Panamá (Herman 1986, 2001; Navarrete-Heredia et al. 2002).

4. Staphylininae, Staphylinini, Philonthina: Belonuchus basiventris (Sharp, 1885) (Fig. 1a). Hidalgo: "México: Hidalgo, Huasca de Ocampo, San Miguel Regla, bosque Las Truchas, bosque de encino, 2073 m, N 20 13'0.3”, W $98^{\circ}$ 33'28.6", en hojarasca cernida, 14-VI-2007, J. Márquez y J. Asiain cols." (1). Registros previos: Estado de México (Nanchititla), Jalisco, Morelos (Cuernavaca, Tlayacapan), Oaxaca (Oaxaca, Yolox), Puebla (Atlixco, Puebla) y Veracruz (Cordoba, Xalapa) (Sharp 1885; Jiménez-Sánchez et al. 2000; Márquez 2001; Navarrete-Heredia et al. 2002; Márquez \& Asiain 2006).

5. Belonuchus bidens Sharp, 1885 (Fig. 2a). San Luis Potosí: "México: San Luis Potosí, Xilitla, Las Pozas de James, N $21^{\circ} 23^{\prime} 55.1^{\prime \prime}$, W 98 58'6.7”, bosque tropical subperennifolio, $585 \mathrm{~m}$, bajo roca a orilla del río, 16-VII-2007, J. Asiain y J. Márquez cols." (1). Registros previos: Chiapas (Tuxtla), Oaxaca, Puebla (Xicotepec de Juárez) y Veracruz (Los Tuxtlas, Mirador, Xalapa); Nicaragua (Sharp 1885; Navarrete-Heredia et al. 2002; Márquez 2004; Márquez \& Asiain 2006).

6. Staphylinina: Platydracus ferox (Nordmann, 1837) (Fig. 3a). San Luis Potosí: "México: San Luis Potosí, Xilitla, Las Pozas de James, N 21ํ23'55.1", W $98^{\circ}$ $58^{\prime} 6.7$ ", bosque tropical subperennifolio, $585 \mathrm{~m}$, trampa de intercepción de 


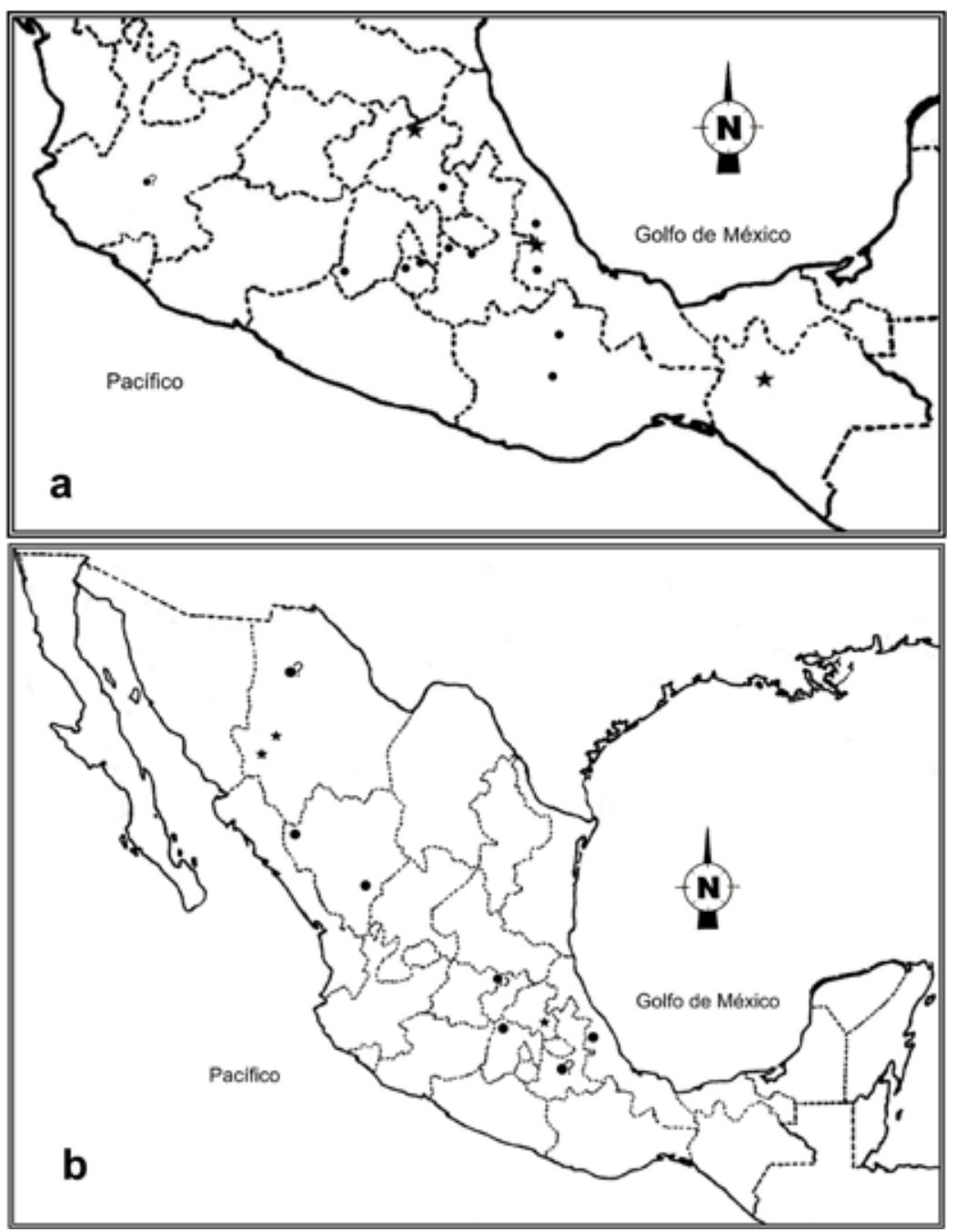

Figura 1. Distribución geográfica de: a, Belonuchus basiventris y $\star$ Pleplomicrus mexicanus; b, $\star$ Deleaster trimaculatus y Lordithon arizonensis.

vuelo, 15 a 17-VII-2007, J. Asiain, J. Márquez, I. A. Rodríguez y A. P. Martínez cols." (1). Registros previos: Campeche, Chiapas, Hidalgo (Tepehuacán de Guerrero), Morelos (Tlayacapan), Oaxaca, Puebla (Coyomeapan, Xicotepec de Juárez), Querétaro, Quintana Roo y Veracruz (Córdoba, El Mirador, Tuxpan, Xalapa); Costa Rica, Guatemala, Nicaragua y Panamá (Navarrete-Heredia et al. 2002; Márquez 2006; Márquez \& Asiain 2006). 

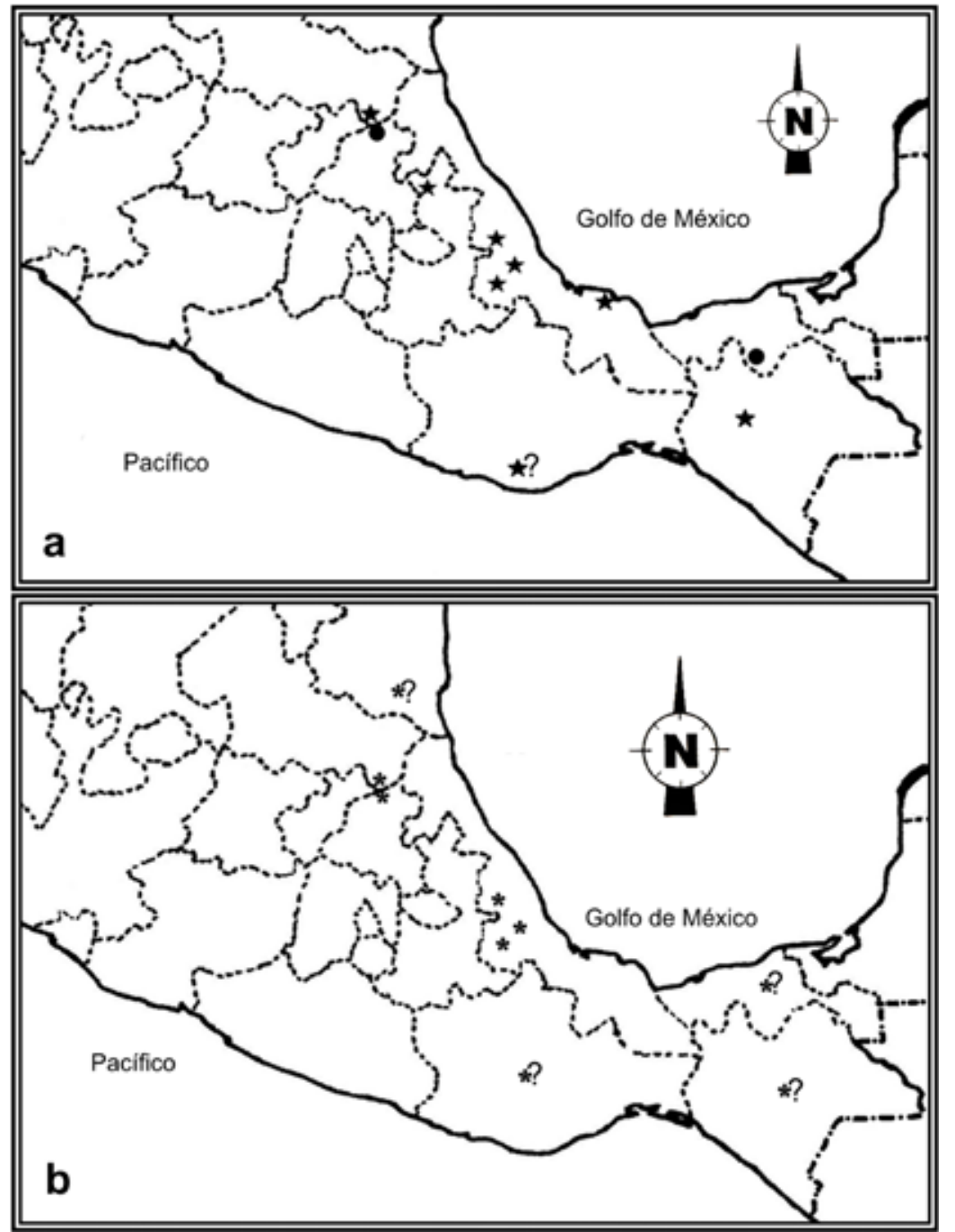

Figura 2. Distribución geográfica de: a, $\star$ Belonuchus bidens y $\bullet$ Bledius inornatus; b, $\boldsymbol{\star}$ Oligotergus fasciatus.

7. Xanthopygina: Oligotergus fasciatus (Nordmann, 1837) (Fig. 2b). Hidalgo y San Luis Potosí: "México: Hidalgo, Chapulhuacán, Arroyo Blanco, selva mediana subperenifolia, $263 \mathrm{~m}, \mathrm{~N} 21^{\circ} 9^{\prime} 37.7^{\prime}$ ', W 990'1.3', trampa de intercepción de vuelo, 1 a 4-VI-2007, J. Márquez y J. Asiain cols." (2). "México: San Luis Potosí, Xilitla, Las Pozas de James, N 21²3'55.1”, W 98 58'6.7”, 


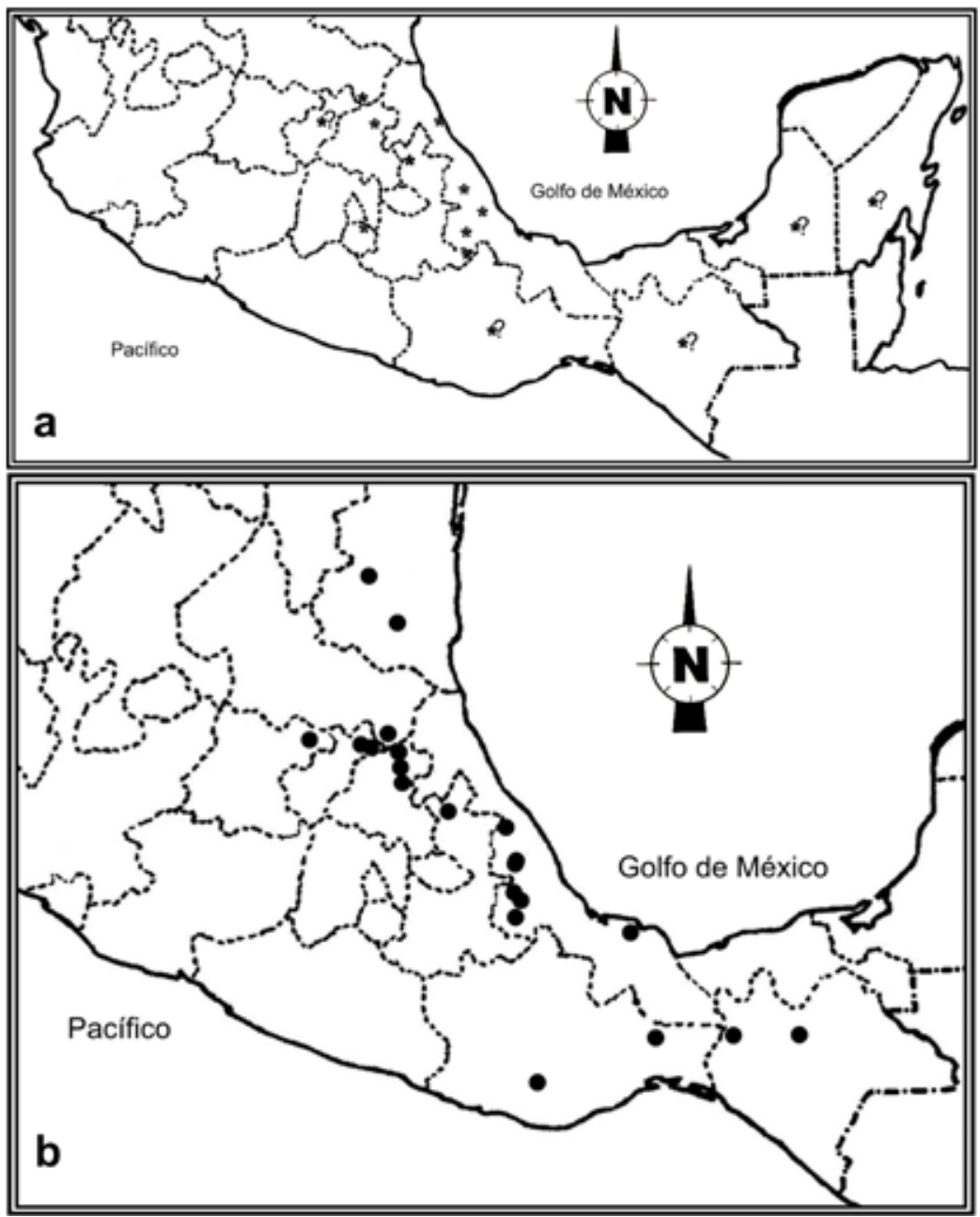

Figura 3. Distribución geográfica de: a, \$ Platydracus ferox; b, - Homalolinus divisus.

bosque tropical subperennifolio, $585 \mathrm{~m}$, trampa de intercepción de vuelo, 15 a 17-VII-2007, J. Asiain, J. Márquez, I. A. Rodríguez y A. P. Martínez cols.” (1). Registros previos: Chiapas, Oaxaca, Tabasco, Tamaulipas y Veracruz (Córdoba, El Mirador, Xalapa); Bolivia, Brasil, Colombia, Costa Rica, Guatemala, Guyana, Panamá, Perú, Trinidad y Venezuela (Navarrete-Heredia et al. 2002; Márquez et al. 2004; Márquez \& Asiain 2006).

8. Plociopterus fetialis (Erichson, 1839) (Fig. 4a). Hidalgo: "Chapulhuacán, Tamaulipas, bosque mesófilo de montaña, $1115 \mathrm{~m}, \mathrm{~N} 21^{\circ} 9^{\prime}$ '56', W 98 55'50.2', 


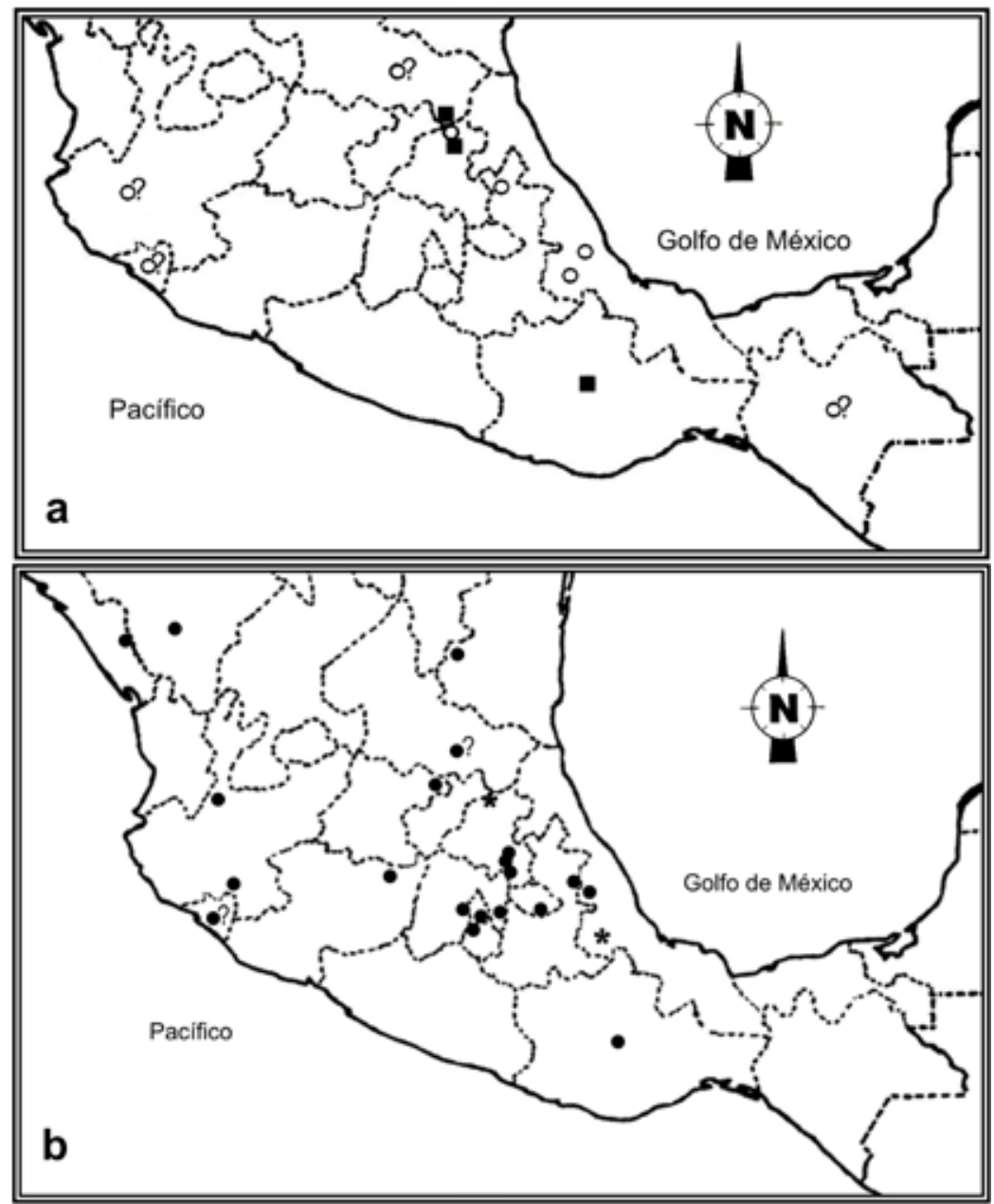

Figura 4. Distribución geográfica de: a, O Plociopterus fetialis y Xanthopygus rufipennis; b, - Tachinus mexicanus y $\boldsymbol{*}$ Tyreocephalus cribripennis.

trampa de intercepción de vuelo, 14 a 17-VII-2007, P. Martínez, J. Asiain, I. Rodríguez y J. Márquez cols." (1). Registros previos: Chiapas, Colima, Jalisco, Puebla (Xicotepec de Juárez), San Luis Potosí y Veracruz (Córdoba, El Mirador); Guatemala y Panamá (Sharp 1884; Navarrete-Heredia et al. 2002; Márquez 2004; Márquez \& Asiain 2006).

9. Xanthopygus rufipennis Sharp, 1884 (Fig. 4a). San Luis Potosí: "México: San Luis Potosí, Xilitla, Las Pozas de James, N 21 23'55.1”, W 98 58'6.7”, bosque tropical subperennifolio, $585 \mathrm{~m}$, trampa de intercepción de vuelo, 15 a 
17-VII-2007, J. Asiain, J. Márquez, I. A. Rodríguez y A. P. Martínez cols.” (1). Registros previos: Hidalgo (Tepehuacán de Guerrero) y Oaxaca (Oaxaca); Guatemala, Nicaragua y Panamá (Sharp 1884; Scheerpeltz 1969; NavarreteHeredia 2004).

10. Xantholinini: Homalolinus divisus (Erichson, 1839) (Fig. 3b). San Luis Potosí: "México: San Luis Potosí, Xilitla, Las Pozas de James, N $21^{\circ}$ 9'52.3", W 98 57'29.6", bosque tropical subperennifolio, $585 \mathrm{~m}$, en tronco con hongos, 18-VII-2007, J. Asiain y J. Márquez cols." (1). Registros previos: Chiapas (El Bosque, Laguna Bélgica, Ocozocoautla, El Ocote), Hidalgo (Chapulhuacán, Tepehuacán de Guerrero, Tlanchinol), Guanajuato (Victoria), Oaxaca (Rizo de Oro, Nizavigueti, Ejuntla), Puebla (Xicotepec de Juárez), Querétaro (Landa de Matamoros, Jalpan, El Lobo), Tamaulipas (Gómez Farías, Hierbabuena, Victoria) y Veracruz (Dos Amates, Córdoba, Fortín de las Flores, Huatusco, Totutla, Xalapa, Coatepec, Teocelo, Tlapacoyan, Julotepec, Naolinco); Costa Rica, Guatemala y Honduras (Márquez 2003, 2006).

11. Thyreocephalus cribripennis Sharp, 1885 (Fig. 4b). Hidalgo: "México: Hidalgo, La Misión, Lomas del Pericón, bosque mesófilo de montaña, 1377 m, N $21^{\circ} 06^{\prime} 46.0^{\prime \prime}, \mathrm{W} 99^{\circ} 06^{\prime} 15.6 ”$, NTP-80 (calamar), 8 a 16-III-2008, J. Márquez y J. Asiain cols. (1). Registros previos: Veracruz (Córdoba) (Sharp 1885; Navarrete-Heredia et al. 2002).

12. Tachyporinae, Mycetoporini: Lordithon arizonensis (Bernhauer, 1912) (Fig. 1b). Guanajuato: "México: Guanajuato, Victoria, Puerto del Aire, km 32, N $21^{\circ} 18.306^{\prime}, \mathrm{W} 100^{\circ} 9.911^{\prime}$, bosque de encino, trampa de intercepción de vuelo, 13 a 16-VII-2009, M. Vargas y J. Márquez cols." (9). "México: Xichú, km 36 después de Puerto del Aire, N 21 18.943', W 100 8.253', bosque de encino reforestado con pino, trampa de intercepción de vuelo, 13 a 16-VII2009, M. Vargas y J. Márquez cols.” (2). Registros previos: Chihuahua, Durango (Canelas, Durango), Estado de México (Acambay), Puebla y Veracruz (Las Vigas); USA (Campbell 1982; Navarrete-Heredia et al. 2002).

13. Tachyporini: Tachinus mexicanus Campbell, 1973 (Fig. 4b). Guanajuato: "México: Guanajuato, Victoria, Puerto del Aire, km 32, N 21 18.306', W $100^{\circ} 9.911$ ", bosque de encino, trampa de intercepción de vuelo, 13 a 16-VII2009, M. Vargas y J. Márquez cols.” (1). Registros previos: Colima, Distrito Federal (Milpa Alta, Xochimilco), Durango (El Salto), Hidalgo (Epazoyucan, Mineral del Chico, Mineral del Monte, Singuilucan, Huasca, Zapotlán), Jalisco (Nevado de Colima y Volcán de Tequila), Estado de México (Amecameca, Parque Nacional Ixta-Popo, Salazar), Michoacán (Puerto Garnica), Morelos (Huitzilac), Oaxaca (Oaxaca), Puebla (Teziutlán), San Luis Potosí, Sinaloa (El Palmito), Tamaulipas (Miquihuana), Tlaxcala (P. N. La Malinche) y Veracruz (Las Vigas); Guatemala y USA (Campbell 1973; Navarrete-Heredia et al. 2002; Márquez 2006; Márquez \& Asiain 2006). 
Siete de los 14 primeros registros son para el estado de Hidalgo, cinco para San Luis Potosí y dos para Guanajuato. Las localidades de colecta de los primeros registros estatales se ubican en las provincias biogeográficas del Golfo de México (7), Sierra Madre Oriental (5) y Eje Volcánico Transmexicano (2) (sensu Morrone 2005).

\section{DISCUSIÓN}

$\mathrm{Al}$ analizar la distribución geográfica de las 13 especies con primeros registros estatales (Figs. 1-4), se aprecia que siete de ellas (Bledius inornatus, Belonuchus bidens, Platydracus ferox, Oligotergus fasciatus, Plociopterus fetialis, Xanthopygus rufipennis y Homalolinus divisus) (Figs. 2a-b, 3a-b, 4a) son de distribución neotropical amplia, abarcando varios estados mexicanos y extendiéndose hacia América Central o hasta América del Sur. Tres especies presentan una distribución restringida al centro (Belonuchus basiventris) (Fig. 1a) y sureste de México (Peplomicrus mexicanus y Thyreocephalus cribripennis) (Figs. 1a y 4b); mientras que Deleaster trimaculatus y Lordithon arizonensis se distribuyen desde la región Neártica (USA y norte de México) hasta algunos estados centrales de la República Mexicana (Fig. 1b). Finalmente, Tachinus mexicanus puede considerarse de distribución amplia (Fig. 4b, Cuadro 1).

Es notable que la mayoría de las especies analizadas en este trabajo presentan distribución neotropical (Cuadro 1), aspecto que coincide con un análisis previo de estafilínidos mexicanos, donde se apreció una mayor influencia neotropical que neártica en este grupo de coleópteros (Márquez \& Asiain 2006). Sin embargo, puede deberse a que se han estudiado ejemplares de la región centro-sur del país y no de los estados del norte, donde puede haber mayor influencia neártica. La distribución de las especies estudiadas corresponde con cuatro patrones biogeográficos, dos de ellos se han considerado componentes bióticos principales, el Mexicano de Montaña y el Mesoamericano (Morrone \& Márquez 2003), el tercero es la combinación del componente Mexicano de Montaña con el Neártico Continental, y el cuarto es un patrón biogeográfico que no se ha propuesto previamente, denominado aquí Sureste de México. Los datos disponibles y el nivel de análisis de este trabajo no permiten proponerlo como componente biogeográfico o con alguna otra categoría homóloga a áreas de endemismo previamente documentadas, simplemente reconocemos este patrón en algunas especies incluidas en este trabajo y otras documentadas en estudios previos.

1. Componente Mexicano de Montaña: incluye las provincias de la Sierra Madre Occidental, Sierra Madre Oriental, Eje Volcánico Transmexicano, Cuenca del Balsas y Sierra Madre Oriental Sur. La distribución de Belonuchus basiventris y varios taxones de coleópteros sustenta la homología biogeográfica primaria entre estas áreas de endemismo (Fig. 1a, Cuadros 1 y 2, referencias en el Cuadro 2). 
Acta Zool. Mex. (n.s.) 28(1) (2012)

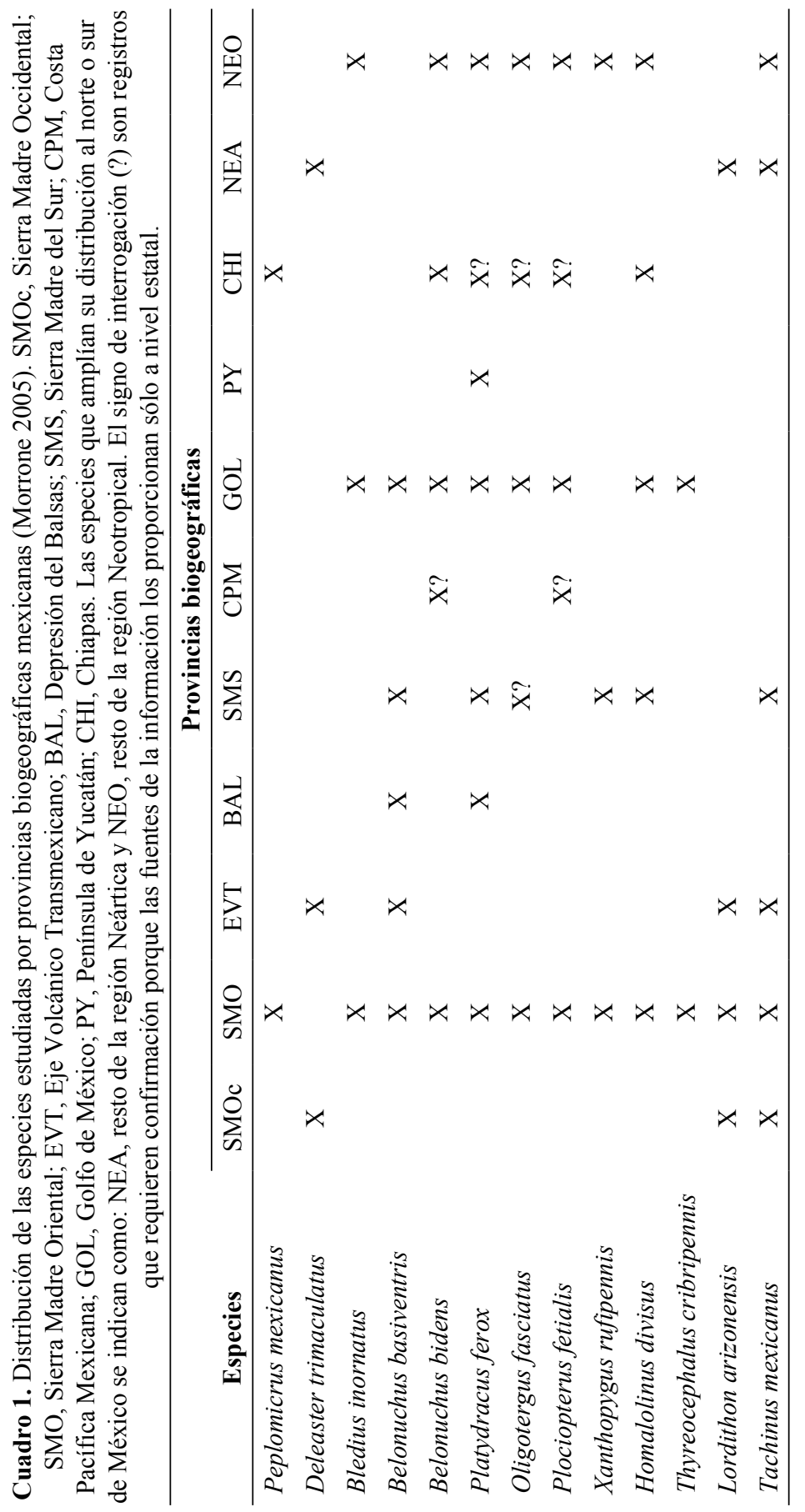

127 


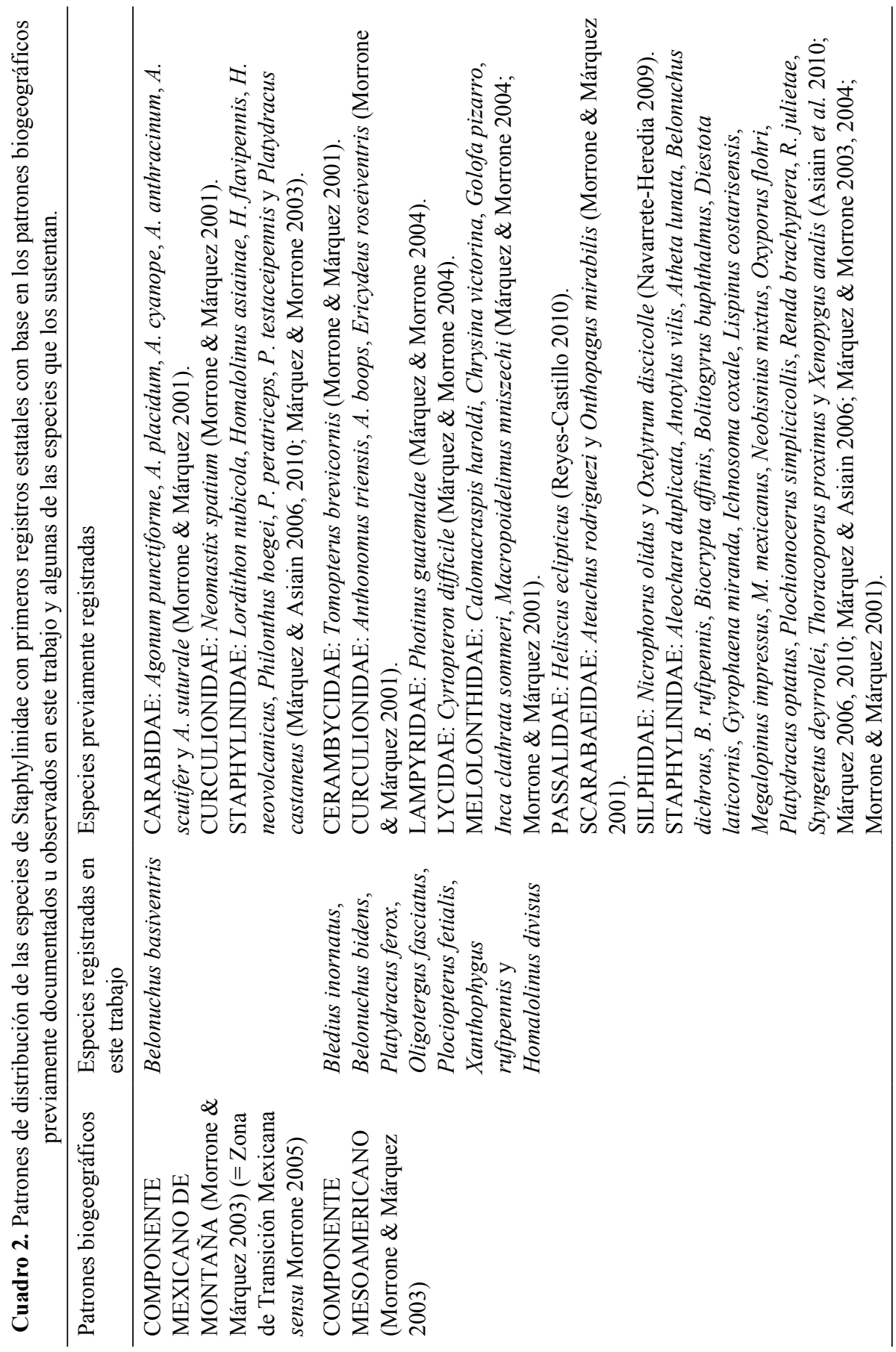




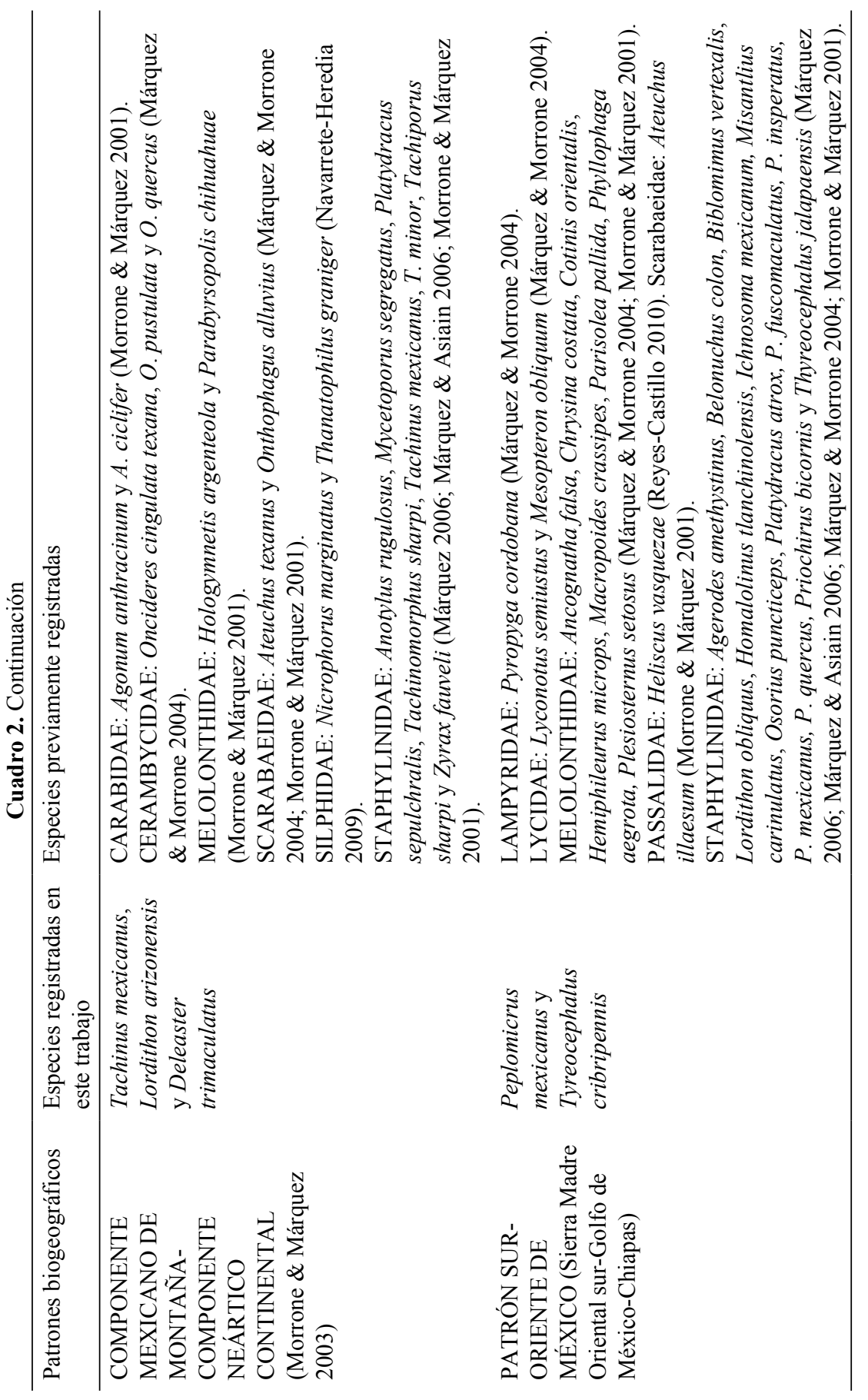


2. Componente Mesoamericano: incluye las provincias del Golfo de México, Costa Pacífica Mexicana y Chiapas, además de extender su distribución hacia el resto de la región Neotropical. Siete de las especies estudiadas soportan este patrón (Figs. 2a-b, 3a-b, 4a; Cuadros 1 y 2). La relación biogeográfica entre estas provincias mexicanas y con el resto de la región Neotropical ha sido sustentada por varios taxones de coleópteros (Cerambycidae, Curculionidae, Melolonthidae, Scarabaeidae, Silphidae y Staphylinidae) (Cuadro 2).

3. Componente Mexicano de Montaña-Componente Neártico Continental: incluye las mismas provincias señaladas para el Componente Mexicano de Montaña más las provincias de Sonora, Altiplano Mexicano y Tamaulipas, extendiéndose hacia los Estados Unidos de América. La distribución geográfica de Tachinus mexicanus, Lordithon arizonensis y Deleaster trimaculatus apoya la probable homología biogeográfica primaria entre estas provincias mexicanas y con el resto de la región Neártica, en adición al sustento de otras especies de diferentes familias de coleópteros, como Carabidae, Cerambycidae, Melolonthidae, Scarabaeidae, Silphidae y Staphylinidae (Figs. 1 b y 4b; Cuadros 1 y 2).

4. Patrón Sureste de México: incluye las provincias de la Sierra Madre Oriental (porción sur), Golfo de México y Chiapas. Peplomicrus mexicanus y Tyreocephalus cribripennis están presentes en estas provincias (Figs. 1a y 4b; Cuadro 1). La probable relación biogeográfica entre estas tres áreas de endemismo ha sido documentada previamente por Márquez \& Morrone (2004), con base en la distribución de diferentes taxones de coleópteros (Lampyridae, Melolonthidae, Passalidae, Scarabaeidae y Staphylinidae) (Cuadro 2). En uno de los análisis de estos autores, encuentran que cuando dividen a la Sierra Madre Oriental en una parte norte (norte de San Luis Potosí a Coahuila) y otra sur (Veracruz a sur de San Luis Potosí), la parte norte se relaciona con el resto de la región Neártica y la parte sur está más relacionada con la provincia del Golfo de México, y éstas dos últimas aparecen relacionadas con la provincia de Chiapas.

Por otro lado, la distribución congruente de especies en estas tres áreas de endemismo (Sierra Madre Oriental sur, Golfo de México y Chiapas) como parte del componente Mesoamericano, ha sido ampliamente documentada con base en el análisis de distintos taxones de coleópteros (Cuadro 2). Sin embargo, existe la posibilidad de que varios de los taxones que han sido documentados para esa área sureste de México sean endémicos a ella, pero también puede tratarse de datos faltantes en cuanto a sus registros en otras áreas de endemismo, que también puede ocurrir en cualquiera de los patrones anteriormente comentados, pero en éste parece ser mas notorio por el menor tamaño del área geográfica que ocupan.

Agradecimientos. A Juan J. Morrone (Facultad de Ciencias, UNAM) por la revisión del manuscrito. A Lee Herman (American Museum of Natural History) por la identificación de una especie. Al proyecto 
FOMIX-CONACyT "Diversidad Biológica del Estado de Hidalgo (segunda fase)", clave HGO-200801-95828, por el apoyo económico brindado para realizar trabajo de campo en el estado de Hidalgo. Agradecemos a dos revisores anónimos por sus valiosos comentarios para concluir este trabajo.

\section{LITERATURA CITADA}

Asiain, J., J. Márquez \& J. J. Morrone. 2010. Track analysis of the species of Agrodes and Plochionocerus (Coleoptera: Staphylinidae). Revista Mexicana de Biodiversidad, 81: 177-181.

Campbell, J. M. 1973. A revision of the genus Tachinus (Coleoptera: Staphylinidae) of North and Central America. Memoirs of Entomological Society of Canada, 90: 1-137.

Campbell, J. M. 1978. New species and records of New World Micropeplidae (Coleoptera). II. Canadian Entomologist, 110: 1247-1258.

Campbell, J. M. 1982. A revision of the genus Lordithon Thomson of North and Central America (Coleoptera: Staphylinidae). Memoirs of the Entomological Society of Canada, 119: 1-116.

Delgado, L. \& J. Márquez. 2006. Estado del conocimiento y conservación de los coleópteros Scarabaeiodea (Insecta) del estado de Hidalgo, México. Acta Zoológica Mexicana (n. s.), 22: 57-108.

Herman, L. 1986. Revision of Bledius. Part IV. Classification of species groups, phylogeny, natural history, and catalogue (Coleoptera, Staphylinidae, Oxytelinae). Bulletin of the American Museum of Natural History, 184: 1-368.

Herman, L. 2001. Catalog of the Staphylinidae (Insecta: Coleoptera). 1758 to the end of the second millennium. Parts III. Oxyteline Group. Bulletin of the American Museum Natural History, 265: 1067-1806.

Jiménez-Sánchez, E., J. L. Navarrete-Heredia \& J. Padilla-Ramírez. 2000. Estafilínidos (Coleoptera: Staphylinidae) necrófilos de la Sierra de Nanchititla, Estado de México, México. Folia Entomológica Mexicana, (108): 53-78.

Márquez, J. 2001. Especies necrófilas de Staphylinidae (Insecta: Coleoptera) del municipio de Tlayacapan, Morelos, México. Folia Entomológica Mexicana, 40: 93-131.

Márquez, J. 2003. Systematic revision of the genera Homalolinus Sharp, 1885 and Ehomalolinus Bierig, 1934 (Coleoptera: Staphylinidae, Xantholinini). Zoologica Scripta, 32: 491-523.

Márquez, J. 2004. Primeros registros estatales de especies mexicanas de Staphylininae (Coleoptera: Staphylinidae). Acta Zoológica Mexicana (n. s.), 20: 91-97.

Márquez, J. 2006. Primeros registros estatales y datos de distribución geográfica de especies mexicanas de Staphylinidae (Coleoptera). Boletín de la Sociedad Entomológica Aragonesa, 38: 181-198.

Márquez, J. 2010. Revision of the genus Renda Blackwelder, 1952 (Coleoptera: Staphylinidae: Xantholinini). Zootaxa, 2686: 1-6.

Márquez, J. \& J. Asiain. 2006. Patrones de distribución de la familia Staphylinidae (Coleoptera), pp. 157-236. In: Morrone, J. J. \& J. Llorente Bousquets (Eds.). Componentes Bióticos Principales de la Entomofauna Mexicana. Las Prensas de Ciencias, UNAM, México, D.F.

Márquez, J. \& J. Asiain. 2010. Three new species of the Philonthus furvus species group (Coleoptera: Staphylinidae) from Guatemala and México, with taxonomic remarks and distributional records of related Mexican species. Transactions of the American Entomological Society, 136: 3-4: 269-288.

Márquez, J., J. Asiain \& Q. Santiago-Jiménez. 2004. Especies de Staphylininae (Coleoptera: Staphylinidae) de "El Mirador", Veracruz, México. Dugesiana, 10: 21-46.

Márquez, J. \& J. J. Morrone. 2003. Análisis panbiogeográfico de las especies de Heterolinus y Homalolinus (Coleoptera: Staphylinidae: Xantholinini). Acta Zoológica Mexicana (n. s.), 90: 15-25.

Márquez, J. \& J. J. Morrone. 2004. Relaciones biogeográficas basadas en la distribución de Coleoptera (Insecta), pp. 375-392. In: Luna, I., D. Espinosa \& J. J. Morrone (Eds.). Biodiversidad de la Sierra Madre Oriental. Las Prensas de Ciencias, Facultad de ciencias, UNAM, México, D.F. 
Morrone, J. J. 2001. Biogeografia de América Latina y el Caribe. M\&T-Manuales \& Tesis SEA, vol. 3, Zaragoza.

Morrone, J. J. 2005. Hacia una síntesis biogeográfica de México. Revista Mexicana de Biodiversidad, 76: 207-252.

Morrone, J. J. \& J. Márquez. 2001. Halffter's Mexican Transition Zone, beetle generalized tracks, and geographical homology. Journal of Biogeography, 28: 635-650.

Morrone, J. J. \& J. Márquez. 2003. Aproximación a un Atlas Biogeográfico Mexicano: Componentes Bióticos Principales y Provincias Biogeográficas, pp. 217-220. In: Morrone, J. J. \& J. Llorente (Eds.). Una perspectiva Latinoamericana de la Biogeografia. Las Prensas de Ciencias, Facultad de Ciencias, UNAM, México, D.F.

Navarrete-Heredia, J. L. 2004 Sinopsis del género Xanthopygus Kraatz, 1857 (Coleoptera: Staphylinidae) de México. Acta Zoológica Mexicana (n. s.), 20: 1-13.

Navarrete-Heredia, J. L. 2009. Silphidae (Coleoptera) de México: Diversidad y distribución. Universidad de Guadalajara, Guadalajara, Jalisco.

Navarrete-Heredia, J. L., A. F. Newton, M. K. Thayer, J. S. Ashe \& D. S. Chandler. 2002. Guía ilustrada para los géneros de Staphylinidae (Coleoptera) de México. Illustrated guide to the genera of Staphylinidae (Coleoptera) of México. Universidad de Guadalajara y CONABIO. Guadalajara, Jalisco.

Navarrete-Heredia, J. L.\& S. Zaragoza-Caballero. 2006. Diversidad de los Staphylinoidea de México: Análisis de grupos selectos (Hydraenidae, Agyrtidae, Silphidae y Staphylinidae). Dugesiana, 13: 53-65.

Reyes-Castillo, P. 2010. Las especies mexicanas de Heliscus Zang, 1906 (Coleoptera: Passalidae). Dugesiana, 17: 193-196.

Sharp, D. 1884. Biologia Centrali-Americana, Insecta, Coleoptera, Staphylinidae. Vol. 1, pt. 2. Taylor \& Francis, London.

Sharp, D. 1885. Biologia Centrali-Americana, Insecta, Coleoptera, Staphylinidae. Vol. 1, pt. 2. Taylor \& Francis, Londron.

Santiago Jiménez, Q. 1999. Los Staphylinidae (Insecta: Coleoptera) necrófilos de un gradiente altitudinal en la región central del estado de Veracruz, México. Tesis, Licenciado en Biología, Universidad Veracruzana, Xalapa.

Scheerpeltz, O. 1969. Die central- und sudamerikanischen Arten der Gattung Xanthopygus Kraatz. Koleopterologische Rundschau, 46/47: 109-118. 